\title{
The relationship between the components of idiopathic focal epilepsy
}

\begin{abstract}
The classification of epileptic syndromes in children is not a simple duty, it requires a great clinical ability as well as some degree of experience. Until today there is no international consensus regarding the different diseases that are part of the Idiopathic Focal Epilepsies However, these diseases are the most prevalent in children suffering from some type of epilepsy, for this reason it is important to know more about these, their clinical characteristics, pathological findings in studies such as encephalogram, resonance, polysomnography and others. In the same way, to explore the possible genetic origins of many of these pathologies, that have been little explored and become an important therapeutic goal. A description of the most relevant aspects of atypical benign partial epilepsy, Landau-Kleffner syndrome, benign epilepsy with centrotemporal spikes, electrical status epilepticus during sleep and Panayiotopoulos syndrome is presented in this review, Allowing physicians and family members of children suffering from these diseases to have a better understanding and establish in this way a better diagnosis and treatment in patients, as well as promote in the scientific community the interest to investigate more about these relevant pathologies in childhood.
\end{abstract}

Keywords: Idiopathic Focal Epilepsies, benign epilepsy, atypical benign partial epilepsy, landau-kleffner syndrome, Rolandic epilepsy, benign epilepsy with centrotemporal spikes, electrical status epileptics during sleep, Panayiotopoulos syndrome, childhood
Volume 8 Issue 3 - 2018

\section{Cesar Ramon Romero Leguizamon}

Department of Drug Design and Pharmacology, University of Copenhagen, Denmark

Correspondence: Cesar Ramon Romero Leguizamon M.D., Department of Drug Design and Pharmacology Faculty of Health and Medical Science, University of Copenhagen, Denmark, Jagtev 160, 2100 Copenhagen, Denmark, Email cesar.leguizamon@sund.ku.dk

Received: February 17, 2018 | Published: May 14, 2018
Abbreviations: ABPE, Atypical benign partial epilepsy; BECTS, Benign epilepsy with centrotemporal spikes; CNVs, Copy number of variations; ESES, Electrical status epilepticus during sleep; EEG, Electroencephalogram; EECSWS, Epileptic encephalopathy with continuous spike-and-wave during sleep; ENM, Epileptic negative myoclonus; IFE, Idiopathic Focal Epilepsies; ILAE, International League Against Epilepsy; LKS, Landau-Kleffner syndrome; NMDA, N-methyl-D-aspartate; PS, Panayiotopoulos syndrome

\section{Introduction}

A way to classify the electro-clinical syndromes is arranged by typical age of onset (neonatal, infancy, childhood, adolescence-adult, familial onset). One of these is the childhood epilepsy, before named as "benign epilepsy", now kwon as Idiopathic Focal Epilepsies (IFE). This group of epilepsies have some similarities between them, either because they share clinical symptoms, encephalographic patterns, alteration in the same gene and/or progress towards the same clinical deterioration. ${ }^{1}$ The main aim of this article is to correlate the commonalities between the Landau-Kleffner syndrome (LKS), electrical status epilepticus during sleep (ESES), atypical benign partial epilepsy (ABPE), benign epilepsy with centrotemporal spikes (BECTS or Rolandic epilepsy), and Panayiotopoulos syndrome (PS). However, the correspondence between these syndromes have been already done, but with controversial findings. ${ }^{2}$ For this reason is that the present review presents the clinical, patho physiological, genetic and complementary studies that help to better understand these syndromes their similarities and differences, to be able to make a better diagnostic approach and thus give a better treatment for affected children for these diseases. The search for information was made by consulting resources bibliographic databases, such as the Science Direct, Scielo, Medline and Pubmed databases, using different combinations of the following descriptors in English: benign epilepsy, Idiopathic Focal Epilepsies, Landau-Kleffner syndrome, electrical status epilepticus during sleep, atypical benign partial epilepsy, benign epilepsy with centrotemporal spikes, Rolandic epilepsy and Panayiotopoulos syndrome. All the articles that could be recovered were reviewed, published until 2017, and those considered relevant were selected.

\section{The idiopathic focal epilepsies members}

\section{Landau-Kleffner syndrome}

a. Historically:The original work made it for Landau and Kleffner and published in 1957 reports the cases of five children with epilepsy and acquired aphasia. The clinical findings of these patients were generalized, partial, myoclonic, or absence seizures, all developed aphasia for different periods of time. In the EEG was evident a paroxysmal disorder, usually bilateral, often more predominant in temporal localization with generalized spikes. ${ }^{3}$ After that in 1992, Paquiet et al, publish the first review of the literature about it. ${ }^{4}$ In 1999 Rossi et al. ${ }^{5}$ and in 2003 Mukhinet al describe the EEG findings and progression in patients with LKS. ${ }^{6}$ In 2001 Robinson et al, demonstrated that children with ESES have no presence of clinical alteration in language capacities in relation with $\mathrm{LKS}^{7}$ and in 2004 McVicar and Shinnar confirmed that clinically the syndrome present a limitation in the language 
abilities compared with the clinical characteristics of other similar epileptic syndromes as ESES. ${ }^{8}$

b. Clinical findings: The children with LKS have a pathological interaction between epilepsy and language, with the major symptom the acquired aphasia. ${ }^{9}$ The patients used to present different types of epileptic attacks such as atypical absences, secondary generalized, pharyngo-oral, atonic falls and hemiconvulsive, at age between the 2 and 10 years, presenting progressive alterations in the receptive and expressive language after the events, additionally have some symptoms as headache and confusion. ${ }^{10}$ When the LKS begins before the 4 years and the duration progress more than 1 year, it is possible to develop alterations in the cognitive and behavioral functions. ${ }^{11}$

c. Electroencephalographic findings: The EEG has a very important role in the diagnosis and evolution of the LKS; regional spikes were observed in all the patients, especially in the fronto-, centro-, or posterior-temporal areas $(67 \%)$ in contrast to CSWS were the discharges have a frontal focus origin $(71 \%) .{ }^{12}$ Also in some LKS patients with behavior disorders have been found frontal lobe discharges $(50 \%){ }^{13}$

d. Genetic characteristics: The genetic and molecular studies of the different types of seizures have been increase during the last 10 years, especially those related with the pathophysiology understanding of the IFE. ${ }^{14}$ In LKS have been establish a genetic/ epigenic origin, related with the identification of copy number of variations $(\mathrm{CNVs}),{ }^{15}$ single mutations and somatic mosaicism. ${ }^{16}$ Studies in LKS patients in a cohort of two discordant monozygotic twin pairs and 11 isolated cases have been identified 16 genes with discordant CNVs. ${ }^{14}$ In relation to the presence of single mutations in childhood focal epilepsies with speech and language dysfunction the GRIN2A is the gen with most strong association. ${ }^{17}$ The somatic mosaicism related with rare variants identified in IFE genes are BSN, COL18A1, GALR1, L2H6DH and RELN. ${ }^{18}$

Electrical status epilepticus during sleep: ESES has been defined as a syndrome that develops during childhood, and its main feature is the appearance on the EEG during non-eye-rapid-movement (Non-REM) sleep, continuous spike waves. The seizures vary in on it presentation, partial motor seizures are more prevalent during the night that appear after the ESES pattern is present, and absence seizures appear during the ESES phase. This electroencephalographic pattern and the seizures usually tend to cease during puberty, and this event usually is joint by the improvement some of the neuropsychological function, thou it has been noted that a full recovery after ESES disappears is very rare. ${ }^{19}$ It is important to bear in mind that ESES merely represents an electrophysiological pattern of 'near-continuous' spike-wave discharges observed in sleep; the accompanying clinical signs and symptoms may differ with different syndromes for example, ESES is seen in both Landau Kleffner syndrome and CSWS - which are two different entities. ${ }^{20}$

History: CSWS was first described by Patry et al. ${ }^{21}$ in six children with epilepsy who had 'subclinical electrical status epilepticus' induced by sleep to designate an EEG finding with subclinical spikes waves (SW) occurring during slow sleep that could last from months to years. ${ }^{21}$ These children did not demonstrate overt clinical manifestations; thus, the condition was described as "subclinical" or "electrical." It was initially described condition as a form of encephalopathy given cognitive impairment in all children, and as more patients were described with this condition, it became clear that neurocognitive deterioration occurred at the same time as the electrical status epilepticus during slow wave sleep developed. Subsequently, the International League Against Epilepsy (ILAE) opted to term the condition "continuous spike and waves during sleep" (CSWS) as a specific epilepsy syndrome characterized by continuous diffuse spike-waves occurring during slow-wave sleep, seen after the onset of seizures, that has a potentially poor outcome despite a noncatastrophic onset of seizures, related to the development of neurocognitive deficits (Commission on Classification and Terminology of the International League Against Epilepsy, 1989). ESES is now the term typically used to describe solely the electrographic findings, while the CSWS syndrome remains a clinical diagnosis of the constellation of neurocognitive deterioration from baseline, affecting motor, language, cognitive, or behavioral development, and a significant proportion of non-REM sleep disrupted by spike-wave discharges. ${ }^{22}$

Clinically: According to ILAE, ESES syndrome is an auto limited disorder that is widely involved with the children ages, its main features are the following (Ribera, 2010): epilepsy with focal crisis and apparently generalized, partial motor seizures, neuropsychological deterioration by causing global or selective cognitive regression, motor deterioration such as ataxia, dyspraxia, dystonia or unilateral deficit and in the EEG patter of spike waves occurring in $85 \%$ of deep sleep and this pattern to persist on three o more EEG records for at least a month The first neurological symptoms usually appear at a medium age of 4.4 years old ${ }^{23}$ while the epilepsy starts at the age of 6.5 years old. ${ }^{24}$ On a 117 patients study it was found that the average time of the persistence of the ESES on the EEG was 10 months, with a range between 2 to 37 months. ${ }^{25}$ Patients that present this pattern have also shown behavioral alterations such as hyperactivity, aggressiveness, depression, hypersomnia, and hemineglect, ${ }^{26}$ abnormalities regarding this behavior has been found in different studies. ${ }^{24,27}$ A recent finding has established a relation between cortical malformations, specifically polimicrogyria and the appearance of ESES, because these patients' neural connections remain normal, however they became horizontal which permits the progression of the electrical impulse faster thus generating the convulsive episode. ${ }^{23,28}$ This condition has been found in numerous studies, such as Caraballo et al, where they found that the appearance of ESES was due to some structural abnormality and the most common abnormality was polimicrogyria. ${ }^{24}$ Neonatal thalamic hemorrhage has been associated as a risk factor to develop ESES, since the thalamus along with the cortex participates in the generation of spike-waves, even though there is still a debate whether it's relative importance and the need of and intact thalamus-cortical circuit to the generation of these discharges. ${ }^{29-31}$

Electroencephalographic findings: The electrical discharges in patients with ESES were predominant on the frontal region, which would explain the behavioral problems ${ }^{32}$, and also discharges in the perysilvian region which are associated with the language impairment ${ }^{33}$ Slow waves during sleep on patients with ESES didn't decrease from the beginning of the sleep until no REM sleep. This finding was predominant of the hemisphere on which the spikewave activity was induced. Despite of this, the decrease of the contra lateral hemisphere was not statistically significant ${ }^{34}$ The mode of the deficit responds to the site of the brain on which the decrease of the slope of the slow waves didn't occur. ${ }^{35}$ The thicker the spike wave was, the more obstruct the decrease of the slope was. ${ }^{35}$ Spike waves discharge during the night and its propagation were associated to hyper metabolism, and some areas with hipo metabolism, probably 
associated with inhibitory functions. Hyper metabolism in ESES has been found in the central region and in the posterior parietal cortex and ind Landau-Kleffner Syndrome on the centrotemporal superior gyrus. ${ }^{36}$

Molecular: The dysfunction of the gen NHE6 (SCL9A6), which transcripts for a endosomal exchanger of $\mathrm{Na}+/ \mathrm{H}+$ it has been related with the predisposition to develop ESES, since this causes alkalosis in the cortex, hence promoting the neural excitability. ${ }^{37}$ Also bio molecular associations regarding CSWS and LKS have been linked with genes that also play an important role in autism such as genetic alterations in regions links ATP13A, CDH9, CDH13, CTNNA3, DIAPH3, MDGA2, SHANK3, which constitute regions that are related with rolandic epilepsy, and/or with impairment in language, and speech and most of those alterations related to autism disorders. ${ }^{38}$ Also number variations in CHRNA7 and PCYT1B was found in patient with ESES however with different clinical characteristics, both related to the cholinergic pathway and the last one associated with the regulation on neuronal sprouting and branching. ${ }^{39}$

Forecast: Just one third of the children with ESES have a long lasting favorable outcome without permanent cognitive impairment. ${ }^{40,22}$ The cognitive impairment has been directly associated with the period of duration of ESES. ${ }^{4}$

\section{A typical benign partial epilepsy}

\section{Definition and clinical features}

ABPE is a special type of epileptic syndrome first described by Aicardi \& Chevrie $^{42}$ studied in the group of IFE, characterized by occurrence of generalized minor, simple focal, generalized tonicclonic se, and rarely myoclonic seizures. That present a typical EEG with focal sharp waves that are identical to those of BECTS. ${ }^{43}$ Because the clinical spectrum exhibits similarities to the LennoxGastaut Syndrome, the term pseudo-Lennox Syndrome was proposed by Doose et al. ${ }^{44}$ Also, according to the EEG findings the ABPE is considered an atypical variant of BECTS..$^{45}$ The onset of seizures occurs between 2 and 6.5 years of age. ${ }^{46}$ Initially a period of typical rolandic seizures is followed by frequent atonic seizures, epileptic negative myoclonus (ENM), partial or generalized motor seizures as well as atypical absence seizures also occur, there is absence of developmental and neurological deficits prior to onset, focal epileptic EEG. abnormality during wakefulness and generalized continuous slow spike-waves during sleep, and favorable seizure and intellectual prognoses. ${ }^{47-49} \mathrm{Hahn}$ et al reported ABPE patients with a delay in language development prior to the onset of epilepsy as well as on patients attending school for mentally handicapped children after remission of seizures. ${ }^{50}$ The ENM includes a brief postural lapse of one or both arms, dropping objects, instability during standing, and inclining of the body or falling down. Relative to the ENM, Akiko et al, found in their study that, performance intelligence quotients, decrease permanently if the period of active negative motor seizures is prolonged, whereas intelligence quotients are maintained if the period of active negative motor seizures is shorter. ${ }^{46}$ Typically the patient with ABEP has therapy resistance that has been associated to the involvement of the frontal cortex in EEG findings. ${ }^{51}$

\section{Electroencephalographic studies}

The electroencephalogram of these patients shows typical bilateral rolandic spikes and spike-wave activity during drowsiness and sleep. Epileptic negative myoclonus is one of the characteristic seizure pattern in $\mathrm{ABPE},{ }^{48}$ this is defined as an interruption (lasting $50-400 \mathrm{~ms}$ ) of tonic muscular activity, time-locked to a spike or to a sharp wave in the area of the contralateral centroparietal cortex on EEG without evidence of a previous myoclonus. ${ }^{52}$ Javadini et al found in a retrospective study that $61 \%$ of patients showed frontal sharp wave foci, that are typical of ABPE..$^{51}$ Also is characteristic the tendency toward generalization while awake or asleep and possibly an extremely irregular pattern similar to that of hypsarrhythmia. ${ }^{43}$

\section{Bio molecular}

Hereditary impairment of brain maturation represents the common basic mechanism shared by BECTS, ABPE, Landau-Kleffner syndrome and EECSWS. ${ }^{43}$ Lemke and collaborators found mutations in gene GRIN2A that encodes the a2 subunit of the N-methyl-Daspartate (NMDA)-selective glutamate receptor that affected patients with the whole spectrum of IFE including ABPE. ${ }^{53}$ Specifically, a missense alteration p. Ala243Val demonstrated impaired reduction of receptor by low concentrations of $\mathrm{Zn} 2+$, suggesting increased activation of the NR1-NR2 Aheteromer due to reduced highaffinity $\mathrm{Zn} 2+$-mediated inhibition in vivo. This results in a higher susceptibility to repeated activation and enhanced $\mathrm{Ca} 2+$ influx. ${ }^{53}$

\section{Benign epilepsy with centrotemporal spikes \\ Definition}

BECTS or Rolandic epilepsy is the most common epileptic syndrome during childhood (children aged 3-13 years). It resolves spontaneously before 15 years of life. "Rolandic" refers to the rolandic area of the brain, which is the part that controls movements. This type of idiopathic and focal epilepsy disappears during adolescence without leave consequences and that is the reason of the term "Benign". Children with BECTS have normal IQ. There is a 1:5 male predominance, prevalence is around $15 \%$ in children aged $1-15$ years with non-febrile seizures and incidence is 10-20/100,000 children aged $0-15$ years. There is an incidence around $14-24 \%$ of newly diagnosed epilepsy in children before 15 years of life. It always begins after 2 years of age (with a peak incidence at age 8 and disappears before 16 years of age.

\section{History}

People believe that Rolandic epilepsy is named due to the anatomical region of the brain, but the real name came from Martinus Rulandus, who gave the first known details of the condition in 1597 based on studies of an abnormal disease in a boy that nobody would classify as a rolandic seizure. In 1941 the electroencephalograph was invented and it was used to identify people with epilepsy. Then, Gastaut noticed the spikes in the pre-rolandic area in children; this information was taken by Nayrac y Beaussart and they established that there was a relation between the seizures described by Rulandus and the spikes in the pre-rolandic area observed on the electroencephalograph, this allowed appearing the name of "Rolandic Epilepsy". Until the 1990s, the principal characteristic of this disease was the electroencephalograph pattern, then Ute Staden (pediatrician, Germany), studied 20 children with BECTS and he noticed that all of them shared a similar way to speak and language. ${ }^{1}$

\section{Clinical}

In rolandic epilepsy seizures are usually brief and occur during sleep or while the child waking up in the morning, are described as 
hemi facial sensor motor manifestations and phonatory accompanied by hemi body motor seizures. The children have tonic deviation of the angle lips, clonic contraction of one side of the mouth and increased salivation with gurgling noises. The episodes last between 1-2 minutes and consciousness and memory of the episode is preserved in $50 \%$ of cases. In more than $50 \%$ of patients seizures occur during sleep and these patients do not present any disability or neuronal injury explaining that they are not usually diagnosed.

The seizure may also involve the throat, which may cause the child's speech to be unclear and therefore difficult to understand. The child may make strange throaty or gurgling noises, and it is often this which alerts parents that something may be wrong. The child often knows what they want to say but cannot speak properly. The seizure can have tonic movements or clonic movements of one side of the face, and then those movements spread to the arm or to the leg, usually on the same side as the movements in the face. Many children lose consciousness, becomes stiff, this is called a tonic-clonic seizure, that is generalized affecting both sides of the brain. After the seizure, many children return to sleep. ${ }^{2}$

\section{Electroencephalographic findings}

The spikes are localized in the $\mathrm{C} 3$ and $\mathrm{C} 4$ or $\mathrm{C} 5$ and $\mathrm{C} 5$ suprasylvian electrodes and not in the temporal electrodes. Centrotemporal spikes are bilateral and activated by slow sleep (non-REM) Centrotemporal spikes are diagnostic markers of BRECTS only when there is a clinical presentation. It is important to know that the frequency, location and persistence do not guide the clinical manifestation, frequency and severity of the seizures. Centrotemporal spikes are not specific of BRECTS as the $2-3 \%$ of normal schoolaged children giant somatosensory evoked spikes. Occur in many types of organic brains disease, for example: cerebral tumours, Rett syndrome, fragile X syndrome, focal cortical dysplasia and others. It is well establish that somatosensory stimulation is one of the factors of activation of centrotemporal spikes and evokes giant somatosensory evoked spikes. The GABA ergic stimulation produces an slow activity on the EEG (10-20\%), so during severe stages of BECTS, cortical inhibition is powerful enough to start the epileptic activity. ${ }^{54}$

\section{Molecular basis}

It's known a genetic susceptibility that tries to explain the etiology, however this genetic susceptibility can't explain all the factors and causes of the syndrome. The gene locus is located on chromosome $15 \mathrm{q} 14$ and it is in charge of encoded for the $\mathrm{KCC} 3 \mathrm{Cl}+/ \mathrm{K}-$ co transporter, essential for the neuronal excitability regulation. In Rolandic epilepsy, this gene is altered causing an extra cellular $\mathrm{K}+$ accumulation and subsequent GAB Aergic inhibition decreasing the inhibitory factor of these cells on the central nervous system. ${ }^{55}$

\section{Panayiotopoulos syndrome}

PS is childhood epilepsy that has a characteristic autonomic symptoms and the crisis occur usually during sleep, that is why often is misdiagnosed with another epileptic syndromes or pathologies or simply remain without a diagnosis. Affects otherwise normal children, the onset is usually at 4 years old, the EEG shows occipital spike-wave paroxysms. The prognosis is excellent; it is self-limited and often does not required antiepileptic drugs. ${ }^{56,57}$

\section{Epidemiology}

Represents the $13 \%$ of all epilepsies in children between 3 and 6 years old, and the $6 \%$ in 1 to 15 years old. ${ }^{58-61}$ The patients may have or not family history of convulsive disorder or febrile convulsions. ${ }^{59,60}$ Approximately $87 \%$ of the cases, the symptoms begins before the age of $8,{ }^{61}$ and $76 \%$ starting between 3 and 6 years. ${ }^{62}$ Is thought that at least 2 to 3 in 1000 children may be affected, and whether cases that are nowadays considered to have atypical features are included, these numbers could be higher. ${ }^{63,64}$ It's considered the most common specific cause of non-febrile, non-convulsive status epileptics in childhood. ${ }^{65}$

\section{History}

The first approach to this syndrome was in 1988-1989 when C. Panayiotopoulos described a new type of epilepsy that he named as "Benign Nocturnal Childhood Occipital Epilepsy", it's main characteristics was define as a ictal triad of nocturnal seizures, tonic deviation of the eyes, and vomiting. ${ }^{66,67}$ But this wasn't the first description of occipital epilepsies, previously in 1982, Gastaut described seizures with visual symptoms mainly or companied by hemiclonic movements, automatisms or headache; the EEG showed high-amplitude spike waves in the occipitoposterotemporal part meanwhile the eyes were closed and in a interictal period; later, the ILAE classified as "Gastaut type of childhood occipital epilepsy". ${ }^{56}$ Panayiotopoulos initially described 24 children with ictal vomiting, 12 of that children had an EEG that showed occipital spikes and another 9 had extraoccipital spike or normal EEG (the other 3 patients had brain damage). ${ }^{66,67}$ Posteriorly ILAE proposed the descriptive term "early onset benign childhood occipital epilepsy", but most authors decided use the eponym "Panayiotopoulos syndrome" ${ }^{68}$ In the ILAE Revised Terminology for Organization of Seizures and Epilepsies 2011 - 2013 was decided eliminated the term "benign". ${ }^{69}$

\section{Clinical presentation}

The beginning occurs usually in the early childhood (3 to 6 years) in the $76 \%$ of the cases. ${ }^{57,58}$ Usually these patients have not a relevant previous familiar or personal history. $17 \%$ have typical febrile seizures. Both sexes are affected in the same way, no ethnic preference. ${ }^{57}$ In the $66 \%$ of the cases the crisis are during sleep, the main symptoms is vomiting $(80 \%)$, and another autonomic manifestations like pallor (28\%), incontinence of urine $(19 \%)$ and faeces $(3 \%)$, hyper salivation $(10 \%)$, cyanosis $(12 \%)$, mydriasis $(7 \%)$, miosis $(2 \%)$, coughing, abnormalities of intestinal motility $(3 \%)$, breathing and cardiac abnormalities could be higher than reported. Cardio respiratory arrest has been reported $(0,4 \%-0,5 \%){ }^{57}$ The consciousness is usually preserved at the beginning of the crisis but lose it posterior. ${ }^{57,58}$ The symptoms can last 5 to 10 minutes, but in the $50 \%$ of the patients reach an autonomic status epileptics (30 minutes or more). ${ }^{57}$ The autonomic symptoms are the main characteristics of the syndrome but not the only ones. There are ictal behavioral changes (agitation, terror, quietness), and non-autonomic symptoms (deviation of the eyes or eyes opening, hemi facial convulsions, visual hallucinations, generalized convulsions, Jacksonian marching) that can occur in combination with other autonomic manifestations. Pure autonomic seizures and pure autonomic status epilepticus is relatively rare, occur in approximately $10 \%$ of patients. They start and terminate usually only with autonomic symptoms..$^{57,58}$

\section{Differential diagnosis}

PS's differential diagnosis have to be made with another pathologies or epileptic syndromes that can be presented with similar symptoms. These are acute encephalitis, migraine, syncope or gastroenteritis and 
structural origins focal epilepsy. ${ }^{58,63}$ Childhood occipital epilepsy of Gastaut and PS are wide different in their symptoms but there are still some physicians that can confound them. Is more common the overlapping with rolandic epilepsy than with other epileptic syndrome.$^{58}$ Like with other syndromes, could exist atypical cases that may be difficult to diagnose.

\section{Prognosis}

The prognosis is excellent, the remission occurs after 1 or 2 years Most of patients present 1 or less than 5 crises until remission. Only $25 \%$ of the cases have very frequent and prolonged seizures. $20 \%$ can develop Rolandic epilepsy or age-related epilepsy that remit. Atypical evolution is rare. The risk for adult epilepsy is the same for the general population. ${ }^{57,70}$ Antiepileptic drugs are usually not need it in a unique crisis, but could be necessary in cases of multiple crises. ${ }^{57}$

\section{Electroencephalographic findings}

The EEG pattern of the PS is usually describe with occipital spikes but the $80 \%$ of the patients have multifocal spikes (parietooccipital, calcarine, central sulci or frontal lobes) but with occipital predominance that can shift from one region to another in the same child $^{58,71}$ Isolated routine EEG is normal in $10 \%$ of the patients, frequently is necessary a continuous sleep recording to detect the

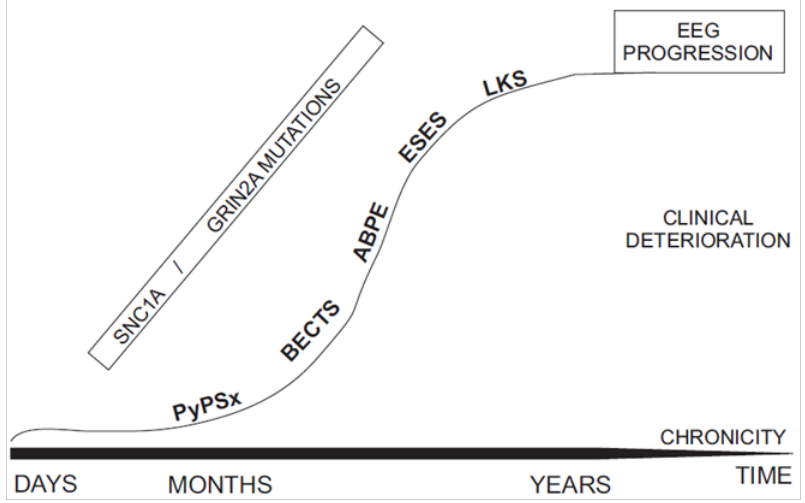

Figure I Integration of the different pathologies that make up idiopathic focal epilepsies, according to their appearance, chronicity, clinical involvement and possible genetic origin.

\section{Conclusion}

The different syndromes that are part of Idiopathic Focal Epilepsies affect a large number of children suffering from some type of epilepsy, for this reason it is important to have an adequate knowledge of them at the moment of making the diagnosis of the patient, in this way can provide the most appropriate treatment for each pathological entity. It is important for the general practitioner that he/she does not have a specialization in pediatric neurology, to be able to establish the differences and similarities that these diseases have, in order to have a positive impact on the prognosis of the patients. Although up to now there is a good amount of information regarding these pathologies, it is necessary to continue with the investigation regarding the possible causes and thus be able to propose new therapeutic alternatives.

\section{Acknowledgements}

None. abnormalities. Sleeps increase the spikes aberrations. The prognosis of the seizures seems to be a favorable despite of EEG pattern. ${ }^{7}$

\section{Genetic characteristics}

Currently there is not a clear etiology of the syndrome, but genetic cause is suspected..$^{70}$ Several studies had been tried to find a specific gene related with PS, but without succeed. One of the suitable genes is SCN1 gene (encodes for alphal subunit of neuronal sodium channel, it has been associated with Dravet Syndrome and generalized epilepsy with febrile seizures plus)..$^{72-74}$ but like Martin del Valle et al suspect that this gene could be not the origin of PS but can be related with its severity. ${ }^{64}$ It's necessary more research in this field (Figure 1). It is important to highlight that these different epileptic syndromes develop in childhood in a different way, and it is essential to determine early the appearance of the symptoms, the importance of a characteristic electroencephalographic tracing and the degree of clinical deterioration that the patient may have. Figure 1 presents a summary of the previous information, showing how the evolution and follow-up of the affected child depends on their clinical evolution, the degree of deterioration in their cognitive functions, as well as the time, allowing to understand which of these syndromes can be more aggressive (despite being called benign) and which of them even have similar genetic origins.

\section{Conflict of interest}

As the author of this article I declare that I do not have any financial interest or any conflict of interest.

\section{References}

1. Pal DK, Ferrie C, Addis L, et al. Idiopathic focal epilepsies: the "lost tribe". Epileptic Disorders. 2016;18(3):252-88.

2. Scheffer IE, Berkovic S, Capovilla G, et al. ILAE classification of the epilepsies: position paper of the ILAE Commission for Classification and Terminology. Epilepsia. 2017;58(4):512-21.

3. Landau WM, Kleffner FR. Syndrome of acquired aphasia with convulsive disorder in children. 1957. Neurology. 1998;51(5):1241 .

4. Paquier PF, Van Dongen HR, Loonen CB. The Landau-Kleffner syndrome or 'acquired aphasia with convulsive disorder'. Long-term followup of six children and a review of the recent literature. Arch Neurol. 1992;49(4):354-359.

5. Rossi PG, Parmeggiani A, Posar A, et al. Landau-Kleffner syndrome (LKS): long-term follow-up and links with electrical status epilepticus during sleep (ESES). Brain Dev. 1999;21(2):90-98.

6. Mukhin K, Kholin AA, Petrukhin AS, et al. Electroclinical characteristics of Landau-Kleffner syndrome. Zh Nevrol Psikhiatr Im S S Korsakova. 2003;103(9):16-27.

7. Robinson RO, Baird G, Robinson G, et al. Landau-Kleffner syndrome: course and correlates with outcome. Dev Med Child Neurol. 2001;43(4):243-247.

8. McVicar KA, Shinnar S. Landau-Kleffner syndrome, electrical status epilepticus in slow wave sleep, and language regression in children. Ment Retard Dev Disabil Res Rev. 2004;10(2):144-149.

9. Billard C, Fluss J, Pinton F. Specific language impairment versus LandauKleffner syndrome. Epilepsia. 2009;50 Suppl 7:21-24.

10. Wang S, Zhang YH, Bao XH, et al. Clinical characteristics and longterm prognosis of Landau-Kleffner syndrome. Zhonghua Er Ke Za Zhi. 2006;44(2):105-109. 
11. Hughes JR. A review of the relationships between Landau-Kleffner syndrome, electrical status epilepticus during sleep, and continuous spikewaves during sleep. Epilepsy Behav. 2011;20(2):247-253.

12. Downes M, Greenaway R, Clark M, et al. Outcome following multiple subpial transection in Landau-Kleffner syndrome and related regression. Epilepsia. 2015;56(11):1760-1766.

13. Nickels K, Wirrell E. Electrical status epilepticus in sleep. Seminars in pediatric neurology. 2008;15(2):50-60.

14. Tsai MH, Vears DF, Turner SJ, et al. Clinical genetic study of the epilepsyaphasia spectrum. Epilepsia. 2013;54(2):280-287.

15. Wang K, Li M, Hadley D, et al. PennCNV: an integrated hidden Markov model designed for high-resolution copy number variation detection in whole-genome SNP genotyping data. Genome Res. 2007;17(11):16651674.

16. Lesca G, Rudolf G, Bruneau N, et al. GRIN2A mutations in acquired epileptic aphasia and related childhood focal epilepsies and encephalopathies with speech and language dysfunction. Nat Genet. 2013;45(9):1061-1066.

17. Turner SJ, Morgan AT, et al. New genes for focal epilepsies with speech and language disorders. Curr Neurol Neurosci Rep. 2015;15(6):35.

18. Conroy J, McGettigan PA, McCreary D, et al. Towards the identification of a genetic basis for Landau-Kleffner syndrome. Epilepsia. 2014;55(6):858865 .

19. Jayakar PB, Seshia SS. Electrical status epilepticus during slow-wave sleep: a review. J Clin Neurophysiol. 1991;8(3):299-311.

20. Veggiotti P, Beccaria F, Guerrini R, et al . Continuous spike-and-wave activity during slow-wave sleep: syndrome or EEG pattern? Epilepsia. 1999;40(11):1593-601.

21. Patry G, Lyagoubi S, Tassinari CA. Subclinical "electrical status epilepticus" induced by sleep in children. A clinical and electroencephalographic study of six cases. Arch Neurol. 1971;24(3):242-252.

22. Singhal NS, Sullivan JE. Continuous Spike-Wave during Slow Wave Sleep and Related Conditions. ISRN Neurol. 2014;2014:619079.

23. Değerliyurt A, Yalnizoğlu D, Bakar EE, et al. Electrical status epilepticus during sleep: a study of 22 patients. Brain Dev. 2015;37(2):250-264.

24. Caraballo RH, Cersósimo RO, Fortini PS, et al. Congenital hemiparesis, unilateral polymicrogyria and epilepsy with or without status epilepticus during sleep: a study of 66 patients with long-term follow-up. Epileptic Disord. 2013;15(4):417-427.

25. Caraballo RH, Veggiotti P, Kaltenmeier MC, et al. Encephalopathy with status epilepticus during sleep or continuous spikes and waves during slow sleep syndrome: a multicenter, long-term follow-up study of 117 patients. Epilepsy Res. 2013;105(1-2):164-173.

26. Yilmaz S, Serdaroglu G, Akcay A, et al. Clinical characteristics and outcome of children with electrical status epilepticus during slow wave sleep. J Pediatr Neurosci. 2014;9(2):105-109.

27. Raha S, Shah U, Udani V. Neurocognitive and neurobehavioral disabilities in Epilepsy with Electrical Status Epilepticus in slow sleep (ESES) and related syndromes. Epilepsy Behav. 2012;25(3):381-385.

28. Galanopoulou AS, Bojko A, Lado F, et al. The spectrum of neuropsychiatric abnormalities associated with electrical status epilepticus in sleep. Brain Dev. 2000;22(5):279-295.

29. Kersbergen KJ, de Vries LS, Leijten FS, et al. Neonatal thalamic hemorrhage is strongly associated with electrical status epilepticus in slow wave sleep. Epilepsia. 2013;54(4):733-740.

30. Monteiro JP, Roulet-Perez E, Davidoff V, et al. Primary neonatal thalamic haemorrhage and epilepsy with continuous spike-wave during sleep: a longitudinal follow-up of a possible significant relation. European Journal of Paediatric Neurology. 2001;5(1):41-47.

31. Blumenfeld H. Cellular and network mechanisms of spike-wave seizures. Epilepsia. 2005;46 Suppl 9:21-33.

32. Scholtes FB, Hendriks MP, Renier WO. Cognitive deterioration and electrical status epilepticus during slow sleep. Epilepsy Behav. 2005;6(2):167-173.

33. Filippini M, Arzimanoglou A, Gobbi G. Neuropsychological approaches to epileptic encephalopathies. Epilepsia. 2013;54 Suppl 8:38-44.

34. Bölsterli BK, Schmitt B, Bast T, et al. Impaired slow wave sleep downscaling in encephalopathy with status epilepticus during sleep (ESES). Clin Neurophysiol. 2011;122(9):1779-1787.

35. Bölsterli Heinzle BK, Fattinger S, Kurth S, et al. Spike wave location and density disturb sleep slow waves in patients with CSWS (continuous spike waves during sleep). Epilepsia. 2014;55(4):584-591.

36. De Tiège X, Trotta N, Op de Beeck M, et al. Neurophysiological activity underlying altered brain metabolism in epileptic encephalopathies with CSWS. Epilepsy Res. 2013;105(3):316-325.

37. Zanni G, Barresi S, Cohen R, et al. A novel mutation in the endosomal $\mathrm{Na}+/ \mathrm{H}+$ exchanger NHE6 (SLC9A6) causes Christianson syndrome with electrical status epilepticus during slow-wave sleep (ESES). Epilepsy Res. 2014;108(4):811-815.

38. Lesca G, Rudolf G, Labalme A, et al. Epileptic encephalopathies of the Landau-Kleffner and continuous spike and waves during slow-wave sleep types: genomic dissection makes the link with autism. Epilepsia. 2012;53(9):1526-1538.

39. Sánchez Fernández I, Loddenkemper T, Peters JM, et al. Electrical status epilepticus in sleep: clinical presentation and pathophysiology. Pediatr Neurol. 2012;47(6):390-410.

40. Liukkonen E, Kantola-Sorsa E, Paetau R, et al. Long-term outcome of 32 children with encephalopathy with status epilepticus during sleep, or ESES syndrome. Epilepsia. 2010;51(10):2023-2032.

41. Kramer U, Sagi L, Goldberg-Stern H, et al. Clinical spectrum and medical treatment of children with electrical status epilepticus in sleep (ESES). Epilepsia. 2009;50(6):1517-1524.

42. Aicardi J, Chevrie JJ. Atypical benign partial epilepsy of childhood. Dev Med Child Neurol. 1982;24(3):281-292.

43. Saadeldin IY, Al-Tala SM. Coexistence of epileptic encephalopathy with continuous spike-and-wave during sleep, atypical benign partial epilepsy, and fixation-off sensitivity in two siblings. Epilepsy Behav. 2011;20(1):116-122.

44. Doose H, Hahn A, Neubauer BA, et al. Atypical "benign" partial epilepsy of childhood or pseudo-lennox syndrome. Part II: family study. Neuropediatrics. 2001;32(1):9-13.

45. Kobayashi S, Inui T, Wakusawa K, et al. A case of atypical benign partial epilepsy with action myoclonus. Seizure. 2013;22(3):242-245.

46. Fujii A, Oguni H, Hirano Y, et al. Atypical benign partial epilepsy: recognition can prevent pseudocatastrophe. Pediatr Neurol. 2010;43(6):411-419.

47. Hirano Y, Oguni H, Osawa M. Epileptic negative drop attacks in atypical benign partial epilepsy: a neurophysiological study. Epileptic Disord. 2009;11(1):37-41.

48. Shiraishi H, Haginoya K, Nakagawa E, et al. Magnetoencephalography localizing spike sources of atypical benign partial epilepsy. Brain Dev. 2014;36(1):21-27. 
49. Moeller F, Moehring J, Ick I, et al. EEG-fMRI in atypical benign partial epilepsy. Epilepsia. 2013;54(8):e103-e108.

50. Hahn A, Pistohl J, Neubauer BA, et al. Atypical "benign" partial epilepsy or pseudo-Lennox syndrome. Part I: symptomatology and long-term prognosis. Neuropediatrics. 2001;32(1):1-8.

51. Japaridze N, Menzel E, von Ondarza G, et al. Risk factors of cognitive outcome in patients with atypical benign partial epilepsy/pseudo-Lennox syndrome (ABPE/PLS) and continues spike and wave during sleep (CSWS). Eur J Paediatr Neurol. 2014;18(3):368-375.

52. Yang Z, Liu X, Qin J, et al. A study on epileptic negative myoclonus in atypical benign partial epilepsy of childhood. Brain \& development. 2009;31(4):274-281.

53. Lemke JR, Lal D, Reinthaler EM, et al. Mutations in GRIN2A cause idiopathic focal epilepsy with rolandic spikes. Nat Genet. 2013;45(9):10671072

54. Massa R, de Saint-Martin A, Carcangiu R, et al. EEG criteria predictive of complicated evolution in idiopathic rolandic epilepsy. Neurology. 2001;57(6):1071-1079.

55. Steinlein OK, Neubauer BA, Sander T, et al. Mutation analysis of the potassium chloride cotransporter KCC3 (SLC12A6) in rolandic and idiopathic generalized epilepsy. Epilepsy Res. 2001;44(2-3):191-195.

56. Capovilla G, Striano P, Beccaria F. Changes in Panayiotopoulos syndrome over time. Epilepsia. 2009;50(suppl 5):45-48.

57. Panayiotopoulos CP, Michael M, Sanders S, et al. Benign childhood foca epilepsies: assessment of established and newly recognized syndromes. Brain. 2008;131(Pt 9):2264-2286.

58. Michael M, Tsatsou K, Ferrie CD. Panayiotopoulos syndrome: an important childhood autonomic epilepsy to be differentiated from occipital epilepsy and acute non-epileptic disorders. Brain Dev. 2010;32(1):4-9.

59. Caraballo R, Cersosimo R, Medina C, et al. Panayiotopoulos-type benign childhood occipital epilepsy: a prospective study. Neurology. 2000;55(8):1096-1100.

60. Oguni H, Hayashi K, Funatsuka M, et al. Study on early-onset benign occipital seizure susceptibility syndrome. Pediatr Neurol. 2001;25(4):312318

61. Degerliyurt A, Teber S, Bektas O, et al. Panayiotopoulos syndrome: a case series from Turkey. Epilepsy Behav. 2014;36:24-32.
62. Dura-Trave T, Yoldi-Petri ME, Gallinas-Victoriano F. Panayiotopoulos syndrome: epidemiological and clinical characteristics and outcome. Eur J Neurol. 2008;15(4):336-341.

63. Covanis A. Panayiotopoulos syndrome: a benign childhood autonomic epilepsy frequently imitating encephalitis, syncope, migraine, sleep disorder, or gastroenteritis. Pediatrics. 2006;118(4):e1237-e1243.

64. Martin Del Valle F, Diaz Negrillo A, et al. Panayiotopoulos syndrome: probable genetic origin, but not in SCN1A. Eur $J$ Paediatr Neurol. 2011;15(2):155-157.

65. Okanishi T, Maegaki Y, Ohno K, et al. Underlying neurologic disorders and recurrence rates of status epilepticus in childhood. Brain development. 2008;30(10):624-628.

66. Panayiotopoulos CP. Vomiting as an ictal manifestation of epileptic seizures and syndromes. J Neurol Neurosurg Psychiatry. 1988;51(11):1448-1451.

67. Panayiotopoulos CP. Benign nocturnal childhood occipital epilepsy: a new syndrome with nocturnal seizures, tonic deviation of the eyes, and vomiting. J Child Neurol. 1989;4(1):43-49.

68. Martinovic Z. The new ILAE report on classification and evidencebased commentary on Panayiotopoulos syndrome and autonomic status epilepticus. Epilepsia. 48(6):1215-1216.

69. International Leage Against Epilepsy I. ILAE Revised Terminology for Organization of Seizures and Epilepsies 2011-20132011.

70. Caraballo R, Cersosimo R, Fejerman N. Panayiotopoulos syndrome: a prospective study of 192 patients. Epilepsia. 2007;48(6):1054-1061.

71. Ohtsu M, Oguni H, Hayashi K, et al. EEG in children with early-onse benign occipital seizure susceptibility syndrome: Panayiotopoulos syndrome. Epilepsia. 2003;44(3):435-442.

72. Livingston JH, Cross JH, McLellan A, et al. A novel inherited mutation in the voltage sensor region of SCN1A is associated with Panayiotopoulos syndrome in siblings and generalized epilepsy with febrile seizures plus. $J$ Child Neurol. 2009;24(4):503-508.

73. Grosso S, Orrico A, Galli L, Di Bartolo R, Sorrentino V, Balestri P. SCN1A mutation associated with atypical Panayiotopoulos syndrome. Neurology. 2007;69(6):609-611.

74. Callenbach PM, van den Maagdenberg AM, Frants RR, Brouwer OF Clinical and genetic aspects of idiopathic epilepsies in childhood. Eur $J$ Paediatr Neurol. 2005;9(2):91-103. 